\title{
138. On Concircular Scalar Fields
}

\author{
By Yoshihiro Tashiro \\ (Comm. by Kinjirô Kunugi, M.J.A., Sept. 13, 1965)
}

In a previous paper [1], the author determined complete Riemannian manifolds admitting a concircular scalar field [1, Theorem 1] and, in particular, those admitting a special concircular scalar field [1, Theorem 2]. If $M$ is an $n$-dimensional Riemannian manifold with metric tensor field $g_{\mu \lambda}$, and we denote by $\left\{\begin{array}{c}{ }_{\mu \lambda} \\ \}\end{array}\right\}$ the Christoffel symbol and by $\nabla$ the covariant differentiation with respect to $\left\{\begin{array}{c}{ }_{\mu \lambda} \\ \mu_{\lambda}\end{array}\right\}$, then a concircular scalar field $\rho$ is by definition a scalar field satisfying the differential equation

$$
\nabla_{\mu} \nabla_{\lambda} \rho=\phi g_{\mu \lambda}
$$

where $\phi$ is a scalar field and will be called the characteristic function of $\rho$. A special concircular scalar field is by definition a concircular one satisfying

$$
\nabla_{\mu} \nabla_{\lambda} \rho=(-k \rho+b) g_{\mu \lambda}
$$

with constant coefficients $k$ and $b$. We shall call $k$ the characteristic constant of $\rho$.

In the present paper we shall show that, if a Riemannian manifold admits functionally independent concircular scalar fields, then they are special concircular scalar fields having the same characteristic constant, and that a concircular scalar field, which is not invariant under an infinitesimal isometry, is also a special concircular one. In this light, we may say that the special concircular scalar field is not so special.

A point is called a stationary or ordinary point of $\rho$ according as the gradient vector field $\rho_{\lambda}=\partial_{\lambda} \rho$ vanishes there or not. We notice that the characteristic function $\phi$ of $\rho$ is a differentiable function of $\rho$ itself in a neighborhood of an ordinary point of $\rho$. We shall first show the following

Lemma 1. If $\rho$ and $\sigma$ are concircular scalar fields functionally dependent of each other, then $\sigma$ is linear in $\rho$ with constant coefficients in a neighborhood of an ordinary point of $\rho$.

Proof. Suppose that $\sigma$ satisfies the equation

$$
\nabla_{\mu} \sigma_{\lambda}=\psi g_{\mu \lambda}
$$

where $\sigma_{\lambda}=\partial_{\lambda} \sigma$. By our assumption, we may put $\sigma_{\lambda}=A \rho_{\lambda}, A$ being a proportional factor. By substituting this into (3) and using (1), we have

$$
\left(\partial_{\mu} A\right) \rho_{\lambda}=(\psi-A \phi) g_{\mu \lambda}
$$


Since the matrix $\left(g_{\mu \lambda}\right)$ is non-singular, it follows that $\partial_{\mu} A=0$ and hence $A$ is a constant. Thus $\sigma$ is of the form

$$
\sigma=A \rho+B \text {. }
$$

Applying Ricci's identity to (1), we obtain the equation

$$
K_{\nu \mu \lambda} \rho_{\kappa}=K_{\nu \mu \lambda \kappa} \rho^{\kappa}=-\frac{d \phi}{d \rho}\left(\rho_{\nu} g_{\mu \lambda}-\rho_{\mu} g_{\nu \lambda}\right),
$$

where $K_{\nu \mu \lambda}{ }^{\kappa}$ is the curvature tensor field of $M$. If $\rho$ is, in particular, a special concircular scalar field, then we have

$$
K_{\nu \mu \lambda \kappa} \rho^{\kappa}=k\left(\rho_{\nu} g_{\mu \lambda}-\rho_{\mu} g_{\nu \lambda}\right) \text {. }
$$

We shall have the following

Theorem 1. If two concircular scalar fields $\rho$ and $\sigma$ are functionally independent of each other, then they are special concircular scalar fields having the same characteristic constant.

Proof. The function $\psi$ in (3) is a differentiable function of $\sigma$ and we obtain the equation

$$
K_{\nu \mu \lambda \kappa} \sigma^{\kappa}=-\frac{d \psi}{d \sigma}\left(\sigma_{\nu} g_{\mu \lambda}-\sigma_{\mu} g_{\nu \lambda}\right)
$$

similar to (4). Contracting (4) with $\sigma^{\lambda}$ and (6) with $\rho^{\lambda}$ respectively and taking account of skew-symmetry of $K_{\nu \mu \lambda \kappa}$ in $\kappa$ and $\lambda$, we have

$$
\left(\frac{d \phi}{d \rho}-\frac{d \psi}{d \sigma}\right)\left(\sigma_{\nu} \rho_{\mu}-\sigma_{\mu} \rho_{\nu}\right)=0
$$

Since $\rho$ and $\sigma$ are functionally independent, we see that the first factor should vanish and $\frac{d \phi}{d \rho}=\frac{d \psi}{d \sigma}$ is equal to a constant, say $-k$. Thus $\phi$ and $\psi$ are linear functions of $\rho$ and $\sigma$ respectively.

Theorem 2. If an n-dimensional Riemannian manifold $M$ admits $n-1$ concircular scalar fields $\rho_{a}(\alpha=1, \cdots, n-1)$ and they are functionally independent, then the manifold $M$ is of constant sectional curvature.

Proof. By means of Theorem 1, we have now the equations

$$
\left[K_{\nu \mu \lambda \kappa}-k\left(g_{\nu \kappa} g_{\mu \kappa}-g_{\mu \kappa} g_{\nu \lambda}\right)\right] \rho_{a}^{\kappa}=0
$$

from equations similar to (5). Since the matrix $\left(\rho_{a}^{\kappa}\right)$ is of rank $n-1$, we can put

$$
K_{\nu \mu \lambda \kappa}-k\left(g_{\nu \kappa} g_{\mu \lambda}-g_{\mu \kappa} g_{\nu \lambda}\right)=L_{\nu \mu \lambda} H_{\kappa},
$$

where $L_{\nu \mu \lambda}$ and $H_{\kappa}$ are proportional factors. However, by virtue of skew-symmetry of the left hand side in $\kappa$ and $\lambda$, the above equation should be equal to zero, that is,

$$
K_{\nu \mu \lambda \kappa}=k\left(g_{\nu \kappa} g_{\mu \lambda}-g_{\mu \kappa} g_{\nu \lambda}\right)
$$

and $M$ is of constant sectional curvature.

In a compact manifold $M$, there exist exactly two stationary points of a concircular scalar field $[1$, Theorem 1, C) $]$. Moreover, the only special concircular scalar field admitted in a compact manifold 
$M$ is one having a positive characteristic constant, and the manifold $M$ is then a spherical space $[1$, Theorem 2, III)]. It follows from these facts that

Theorem 3. If a compact Riemannian manifold $M$ admits functionally independent concircular scalar fields, then $M$ is a spherical space.

Next let a vector field $v^{\kappa}$ be an infinitesimal isometry and denote by $£$ the Lie differentiation with respect to $v^{\kappa}$. Then we know the equations

and

$$
£ g_{\mu \lambda}=\nabla_{\mu} v_{\lambda}+\nabla_{\lambda} v_{\mu}=0
$$

$$
£\left\{{ }_{\mu \lambda}{ }_{\mu \lambda}\right\}=\nabla_{\mu} \nabla_{\lambda} v^{\kappa}+v^{\nu} K_{\nu \mu \lambda^{\kappa}}=0
$$

and that $£$ commutes with the covariant differentiation $\nabla$. We shall prove the following

Theorem 4. If a concircular scalar field $\rho$ is not invariant under an infinitesimal isometry $v^{\kappa}$, that is, $£ \rho \neq 0$, then $\rho$ is a special concircular scalar field.

Proof. Applying $£$ to (1), we have

$$
\nabla_{\mu} \nabla_{\lambda} £ \rho=(£ \phi) g_{\mu \lambda}
$$

and see that $£ \rho$ is also a concircular scalar field. If $£ \rho$ is functionally independent of $\rho$, then the theorem follows from Theorem 1. If $£ \rho$ is functionally dependent of $\rho$, then we can put

$$
£ \rho=v^{\kappa} \rho_{\kappa}=A \rho+B
$$

by means of Lemma 1 . Differentiating covariantly (10) and taking account of (1), we have

$$
\left(\nabla_{\lambda} v^{k}\right) \rho_{\kappa}+\phi v_{\lambda}=A \rho_{\lambda}
$$

and, further differentiating (11) and taking account of (7), (8), and (1),

$$
-v^{\nu} K_{\nu \mu \lambda \kappa} \rho^{\kappa}+\left(\partial_{\mu} \phi\right) v_{\lambda}=A \phi g_{\mu \lambda} \text {. }
$$

By contraction of this equation with $\rho^{\lambda}$ and substitution of (10), we obtain

$$
\left(\partial_{\mu} \phi\right)(A \rho+B)=A \phi \rho_{\mu}
$$

The solution of this equation is given by the form

$$
\phi=-k \rho+b
$$

with constant coefficients $k$ and $b$. Thus $\rho$ is a special concircular scalar field.

Q.E.D.

By the same reason as that for Theorem 3, we have

Theorem 5. If a compact Riemannian manifold $M$ admits an infinitesimal isometry $v^{\kappa}$ and a concircular scalar field which is not invariant under $v^{\kappa}$, then the manifold $M$ is a spherical space. 


\section{Reference}

[1] Y. Tashiro: Complete Riemannian manifolds and some vector fields. Trans. Amer. Math. Soc., 117, 251-275 (1965). 